ks. Michael Stickelbroeck

\title{
Die deutschsprachige Mariologie seit der Jahrtausendwende - eine Bestandsaufnahme
}

\section{Inhaltliche Perspektiven}

\section{Zwei Mariologien im NT?}

\subsection{Historisch gegen spekulativ}

Der interessierte Leser begegnet oftmals der These, es gebe im NT zwei Mariologien: eine solle auf historischen Fakten beruhen, die andere, spätere, auf theologischer Reflexion. Als Paradebeispiel einer frühesten Schicht der Mariologie, der des MkEvangeliums, wird Mk 3,20-35 angeführt. In dieser Perikope werden die Verwandten Jesu als unverständig wie die Schriftgelehrten, die ihn für besessen halten, angesehen. Manche Theologen unterscheiden hier nicht zwischen Maria und den übrigen Verwandten und gehen davon aus, dass die Versuchung, Jesus nicht als den Messias zu glauben und außen vor zu bleiben (Mk 3,31: $\square \xi \omega$ ), auch an den Jüngern und den Verwandten und somit auch an Maria nicht vorbeigegangen sei. ${ }^{1}$ Jedoch wird vom Evangelisten nicht gesagt, dass Maria sich mit der Haltung und

1 Vgl. K.-H. Menke, Fleisch geworden aus Maria. Die Geschichte Israels und der Marienglaube der Kirche, Regensburg 1999, 62. 
dem Ausspruch der ungläubigen Verwandten identifiziert hätte: „Er ist von Sinnen“.

K.-H. Menke begegnet dieser Ansicht mit folgender Anfrage: „Kann man aber schon deshalb behaupten, diese ,negative Mariologie' sei die auf Fakten gegründete, im Unterschied zu der auf theologischer Deutung basierenden?" Gegen diese Behauptung macht er geltend, dass die gerade angeführte Perikope konstitutiver Bestandteil eines theologischen Konzeptes bei Mk ist: des Nichtverstehens Jesu nämlich, das sich gerade an der Schlüsselstellte Mk 3,12 zeigt: „auf dass sie sehend sehen und doch nicht schauen, und hörend hören und doch nicht verstehen...". Von daher ist es abwegig, diese "negative Mariologie“ für die auf Fakten beruhende zu halten und gegen eine spätere, die auf theologischer Reflexion beruht, auszuspielen. Man muss auch in Betracht ziehen, dass die beiden Evangelien, die ein Kindheitsevangelium bieten (Mt und Lk) und für ein entwickeltes Stadium einer hochtheologischen, a-historischen Mariologie gehalten werden, die von Mk geschilderte Episode nicht übergehen.

Als weiteres Argument für die Unterscheidung einer historisch zuverlässigen und einer angeblich auf bloßer Spekulation beruhenden Mariologie wird von deren Verfechtern die Aufzählung der namentlich genannten „Brüder“ Jesu in Mk 6,3 ins Feld geführt. Ich gehe hier nicht auf einzelne Argumente ein und verweise nur darauf, dass A. Ziegenaus in seiner Mariologie, wo er Mk 6,3 mit Mk 15,40/Mt 27,55f. parallelisiert, überzeugend nachweist, dass die Existenz von leiblichen Brüdern Jesu in einer sorgfältigen Exegese des NT keinen Anhalt findet. ${ }^{2}$ Anders als die Tradition der katholischen Kirche, die die genannten Brüder Jesu als Vettern betrachtet, sieht die Ostkirche, die sich auf eine im zweiten Jahrhundert entstandene Überlieferung (Protoevangelium des Jakobus) beruft, die Brüder Jesu als Söhne des hochbetagten Joseph aus einer früheren Ehe an.

2 Vgl. A. Ziegenaus, Maria in der Heilsgeschichte. Mariologie (Katholische Dogmatik V), Aachen 1998, 250-259. 
Die meisten Theologen des Protestantismus vertreten die schon von Irenäus bekämpfte These, es handle sich dabei um die nachgeborenen Söhne aus der Ehe Josephs mit Maria. Die in den Kindheitsevangelien hervortretende parthenogenetische Geburt Jesu wird von jenen, die ein Gegeneinander von negativer (historischer) Mariologie bei Mk und positiver (spekulativer) Mariologie bei Mt/Lk vertreten, als ein Eckpunkt der ersteren betrachtet. Es ist jedoch fraglich, ob aus dem Fehlen eines Kindheitsevangeliums bei Mk auf eine solche Opposition geschlossen werden kann.

\subsection{Die Parthenogenesis - nur ein Theologoumenon?}

Theologen wie Anton Vögtle ${ }^{3}$ halten die jungfräuliche Empfängnis Jesu, die sie als „Theo-logoumenon“ bezeichnen, für eine Deutung ohne faktisches Fundament. Vögtle geht von der allgemein angenommenen Hypothese aus, die jungfräuliche Empfängnis Jesu sei zunächst nur im engsten Jüngerkreis geglaubt, nach außen hin aber als Geheimnis bewahrt und selbst nach der Auferstehung Jesu nur ganz allmählich bekannt gemacht worden. Dagegen setzt der Autor die folgende Überlegung, aus der er die eben genannte Konsequenz zieht, dass es sich um ein bloßes Theologoumenon handle: „Die Geltendmachung äußerster Zurückhaltung läßt man sich [...] noch für die Zeit des öffentlichen Wirkens Jesu gern gefallen. Aber auch für die Zeit des nachösterlichen Messiasglaubens? Warum eigentlich ,äußerste Zurückhaltung' und Beschränkung auf ,kleine Kreise'? Etwa weil der Gedanke der geistgewirkten Empfängnis des Messias für jüdisches Empfinden völlig befremdend, ein nicht zumutbares Paradoxon war?"4

3 Vgl. A. Vögtle, Offene Fragen zur lukanischen Geburts- und Kindheitsgeschichte, in: ders., Das Evangelium und die Evangelien, Düsseldorf 1971, 43-56.

4 Ebd., 50. 
Nach der von Vögtle vertretenen Falschmeinung wird der Gedanke der jungfräulichen Empfängnis Jeus die „Abstiegschristologie" bei Paulus mit einer doketischen Nuance belasten.

Doch das Gegenteil ist richtig: Die frühen Väter verwenden die Aussage der Parthenogenesis nicht nur als Zentralargument gegen den Adoptianismus, sondern auch gegen den Doketismus. ${ }^{5}$ Aufgrund der Einheit von Wort (Medium) und Mittler der Offenbarung gab es nicht einen von Joseph gezeugten und aus Maria geborenen Menschen Jesus als Instrument der Offenbarung, sondern das Wort selbst ist Mensch geworden. Daher erfolgte seine Selbstmitteilung nicht an einen schon existierenden Menschen, sondern so, dass Jesus von Anfang an in seinem Menschsein durch die Aufnahme in die Subsistenz des Sohnes Gottes konstituiert wurde. Von daher steht die Parthenogenesis in ihrer biologischen Konkretion für die nicht über ein externes Instrument vermittelte, sondern unmittelbare Menschwerdung.

K.-H. Menke sieht sich in seinen Überlegungen zu diesem Thema veranlasst, zwischen dem Sachgrund und Erkenntnisgrund der Parthenogenesis zu unterscheiden: Es wird von Vögtle u.a. gesagt, die Behauptung der Menschwerdung des Sohnes Gottes als parthenogenetisch sei nur dann kein bloßes Theologoumenon, wenn es dafür eine historisch zuverlässige Tradition gebe. Eine aus früher historischer Tradition gewonnene Überzeugung von der jungfräulichen Empfängnis Jesu (die es faktisch nicht gibt) wird der Alternative „Deutung ohne Faktum“ disjunktiv gegenübergestellt, wobei beides auf der Ebene des Erkenntnisgrundes liegt. ${ }^{6}$

5 Vgl. H. Schürmann, Die geistgewirkte Lebensentstehung Jesu, in: W. Ernst/K. Feiereis (Hrsg.), Einheit in Vielfalt (FS H. Aufderbeck), Leipzig 1974, 156-165.

6 Vgl. K.-H. Menke, Fleisch geworden aus Maria, 66, Anm. 94. Nach Menke tritt diese Alternative in fast allen einschlägigen Beiträgen der deutschen und angelsächsischen Exegese hervor. Er nennt dafür als Beispiel die Autoren: J. Michel, H.J. Brosch/J. Hasenfuss, O. Knoch, K.S. Frank, 
Demgegenüber wäre der eigentliche Sachgrund der Parthenogenesis des Erlösers in Anschlag zu bringen: Das von der Kenosis-Christologie in Gal 4,4, Phil 2,6-11 oder Röm 1,3 genauso wie von den sogenannten "Kindheitsgeschichten“ bei Mt und Lk hervorgehoben Ereignis der Inkarnation. Es könnte doch sein, so Menke, dass die fortschreitende Reflexion dieses Ereignisses im Licht der Offenbarung den wahren Erkenntnis-Grund für die Behauptung der Parthenogenesis lieferte.

\section{Maria - Mittlerin: Mariens mütterliche Mittlerschaft in Christus}

In der Frage der Teilhabe der Gottesmutter an der universalen Mittlerschaft Christi hat der Diskurs unter den Mariologen zu einem beachtlichen Fortschritt geführt. ${ }^{7}$ Schon Paul VI. sah im 8. Kapitel von Lumen Gentium den "Gipfel" und die "Krönung" der Dogmatischen Konstitution über die Kirche. ${ }^{8}$ Sein geistiges Zentrum findet dieses Culmen in der Lehre von der Mitwirkung Marines bei der Erlösung, die sich zur Teilhabe an der Mittlerschaft Christi gestaltet. ${ }^{9}$ Manfred Hauke hat verschiedentlich darauf aufmerksam gemacht, wie sehr die neuer Diskussion angeregt worden ist durch die Initiativen des belgischen Kardinals

F.J. Steinmetz, R. Pesch, K.H. Schelkle, R.E. Brown mit ihren Beiträgen über die Jungfrauengeburt.

7 Vgl. M. Hauke, Maria als mütterliche Mittlerin in Christus. Ein systematischer Durchblick, in: Sedes Sapientiae. Mariologisches Jahrbuch 12 (2008) Bd. 2, 12-41, hier: 13, Anm. 1; vgl. ders., Die mütterliche Vermittlung, in: A. Ziegenaus (Hrsg.), Totus Tuus. Maria in Leben und Lehre Johannes Pauls II. (Mariologische Studien, 18), Regensburg 2004, 125-175.

8 Paul VI., Ansprache Post duos menses zum Abschluss der dritten Konzilsperiode, 21.11.1964: AAS 56 (1964) 1007-1018, hier: 1014.

9 Vgl. M. Hauke, Maria als mütterliche Mittlerin, 16. Die Mittlerschaft Mariens in der Lehre des II. Vatikanums behandelt ders., Mütterliche Vermittlung, 127-130. 
Mercier, mit denen er auf die Dogmatisierung der universalen Gnadenmittlerschaft Mariens abzielte. ${ }^{10}$ Die von Mercier favorisierte Bewegung zugunsten eines neuen marianischen Dogmas gelangte in der Zeit unmittelbar vor dem Zweiten Vatikanum, als hunderte von Bischöfen um eine solche Definition baten, zu ihrem Höhepunkt. Das Konzil hat dann, von anderen Interessen geleitet, die allgemeine Gnadenmittlerschaft Mariens zwar nicht geleugnet, aber doch mit Zurückhaltung behandelt. Deutliche Aussagen zum Thema ergeben sich vor allem aus den Fußnoten. ${ }^{11}$

Erst die Enzyklika Redemptoris Mater Johannes Pauls II. (1977) rückte das Thema der Mittlerschaft Mariens wieder in das Zentrum der Mariologie. Die „mütterliche Mittlerschaft" vollzieht sich nach der Aussage der Enzyklika „in“ Christus.

Der Gebrauch des Terminus „Mittlerin“ setzt eine Rückversicherung über die analoge Sprechweise voraus: Es gibt verschiedene Weisen der Teilhabe. Bei der Mittlerschaft Mariens geht es um ihre Mitwirkung am Erlösungswerk selbst (objektive Erlösung) und um ihre himmlische Fürsprache, durch welche sie auf einzelne Menschen Gnaden herabzieht (subjektive Erlösung) und ankommen lässt. Die mit den Eingaben Merciers anhebende Diskussion hat zum Ergebnis geführt, dass die Mittlerschaft Mariens bereits durch ihre Mitwirkung an der Erlösung beginnt. ${ }^{12}$

Eine Position, die das Mitwirken Mariens auf ein „Empfangen" der Heilsfrüchte (Heinrich Köster) reduziert, muss als überholt gelten, weil das Konzil selbst von einer freiwillentlichen Aktivität der Gottesmutter spricht. Maria wurde „nicht bloß passiv

${ }^{10}$ Vgl. M. Hauke, Maria - die „Mittlerin aller Gnaden“. Die universale Gnadenmittlerschaft Mariens im theologischen und seelsorglichen Schaffen von Kardinal Mercier (1851-1926) (Mariologische Studien, 17), Regensburg 2004.

11 Vgl. Lumen Gentium, 62, Anm. 186-187.

12 Vgl. M. Hauke, Maria als mütterliche Mittlerin, 17, Anm. 11; vgl. ders., Maria - die „Mittlerin aller Gnaden“, 156-177. 
benutzt“, sondern hat „in freiem Glauben und Gehorsam zum Heil der Menschen mitgewirkt" ${ }^{13}$ Die Inkarnation, die durch das Jawort Mariens mit zustande kam, diente nicht nur der Vorbereitung des Erlösungswerkes, sondern gehört selbst mit dazu. Durch ihr Jawort bei der Menschwerdung und ihren consensus unter dem Kreuz, der eine compassio war, wird der besondere Charakter ihrer Mittlerschaft hervorgehoben: „Indem sie Christus empfing, gebar und nährte, im Tempel dem Vater darstellte und mit ihrem am Kreuz sterbenden Sohn litt, hat sie beim Werk des Erlösers in durchaus einzigartiger Weise mitgewirkt zur Wiederherstellung des übernatürlichen Werkes der Seelen “ ${ }^{14}$

Das Konzil hat zwar keine theologischen Kontroversen entscheiden wollen ${ }^{15}$, aber seine Aussagen zur Mitwirkung Mariens an der Erlösung selbst sind hochrangig und wenigstens in diesem Punkt klar. Sie werden nicht ernsthaft in Frage gestellt, sondern gehören zum Konsenz (sententia communis). ${ }^{16}$ Über die bloße Textbasis hinaus ist hier entscheidend die Rezeption der Aussagen von Lumen Gentium, die vom Hauptredaktor Philips, aber auch von Balic, der der zweite Redaktor des Marienkapitels war, sowie von Roschini als eindeutiger Beleg für eine wirkliche Mitwirkung Mariens am objektiven Erlösungswerk gewertet werden. ${ }^{17}$

13 Vgl. Lumen Gentium, 56.

14 Lumen Gentium, 61.

15 Vgl. Lumen Gentium, 54.

16 Die Diskussion um den Titel der „Miterlöserin“ sollte formal vom Thema der Mitwirkung abgehoben werden. Sie stellt ein eigenes Thema dar. Es fehlt dabei oftmals die explicatio terminorum. Außerdem wirken in die Diskussion die Pseudo-Mariengerscheinungen von Amsterdam sowie die Zurückweisung eines Bemühens zugunsten eines weiteren Dogmas hinein. Die Mitwirkung Marines wird in der Regel selbst von jenen, die gegen den Titel „corredemptrix“ polemisieren, nicht in Frage gestellt.

17 Zum status quaestionis vgl. M. Hauke, Maria, "Gefährtin des Erlösers“ (Lumen Gentium, 61). Die Mitwirkung Mariens bei der Erlösung als For- 
Ich sage dies ungeachtet dessen, dass die hyperökumenische "Studie von Dombes", an der auch katholische Theologen mitgewirkt haben, die „Mitwirkung" Mariens in Anführungszeichen setzen. ${ }^{18}$ An erster Stelle erwähnt das Dokument unter den „umstrittenen Fragen“ in der Mariologie „Das ,Mitwirken“ Marias am Heil und die Beziehung zwischen Gnade und Freiheit". ${ }^{19}$ Man zitiert als Beispiel für die protestantische Auffassung Karl Barth: „Er wendet sich vor allem gegen das ,Mitwirken' Marias“. ${ }^{20}$ Die Autoren zitieren gemeinsam Martin Luther: die Rechtfertigung geschieht nicht durch unsere Werke; die Werke offenbaren lediglich das von Gott geschenkte Heil. ${ }^{21}$ Maria ist nur ein Beispiel für das, was in allen Erlösten geschieht. Der Mensch ist passiv gegenüber der Gnade. ${ }^{22}$

In die gleiche Richtung gehen Äußerungen, wie man sie vornehmlich im Internet findet (nicht unter Fachmariologen), die der vor dem Konzil kolportierten Linie von Lennerz entsprechen und jede Mitwirkung am objektiven Erlösungswerk ablehnen. Der Grund dafür besteht in einer ausschließlichen Fokussierung auf das Kreuz, wobei die Inkarnation in ihren Implikationen

schungsthema, in: Sedes Sapientiae 6 (2002) 85-121.

18 Groupe de Dombes, Maria in Gotts Heilsplan und in der Gemeinschaft der Heiligen, Frankfurt a.M. / Paderborn 1999 (frz. 1997 / 98). Vgl. dazu S.M. Perella, La recezione del Documento di Dombes su Maria. Ricognizione bibliografica, Marianum 62 (2000) 347-355.

19 Ebd., nr. 204-227.

20 Ebd., nr. 208.

${ }^{21}$ Vgl. ebd., nr. 216-217.

22 Zur Diskussion darüber vgl. M. Hauke, Maria, Gefährtin des Erlösers, 111-114, hier 112: „Man wird der Gruppe von Dombes als Verdienst anrechnen dürfen, das Thema der marianischen Mitwirkung in die erste Reihe der Tagesordnung für den ökumenischen Dialog gestellt und mit dem grundlegenden Problem der Rechtfertigung verbunden $\mathrm{zu}$ haben. Es hat allerdings nicht den Anschein, dass ein wirkliches Übereinkommen erfolgt ist über die Tatsache, dass, Gott die menschliche Mitwirkung im Rechtefertigungsgeschehen einschließt und nicht ausschließt“. 
kaum bedacht wird. Im allgemeinen generiert eine uneinheitlich gefasste Soteriologie mit ihren verschiedenen Konzeptionen vom Erlösungswerk Jesu eine Lähmung im Hinblick auf das Durchdenken der Mariologie. ${ }^{23}$

Manche Tendenzen, die vom generellen Konsens abweichen, respektieren zwar Lumen Gentium mit seiner Marienlehre, begrenzen jedoch - entgegen des Aussagen des Konzils - die Heilswirkung Mariens de facto auf die subjektive Erlösung. Vorreiter für die von ihnen herkommende Richtung sind Semmelroth und Köster, nach deren Dafürhalten die Mitwirkung Mariens an der objektiven Erlösung bloß rezeptiv ist: Maria nimmt unter dem Kreuz die Erlösungsganden stellvertretend für alle in Empfang. Eine Mitwirkung im eigentlichen Sinn wird damit ausgeschlossen. ${ }^{24}$

Man kann freilich in der Theologie nicht bei den Fußnoten zum II. Vatikanum stehenbleiben. Eine weitere Entwicklung der Lehre haben wir, wie Hauke betont, in den Katechesen Johannes Pauls II., deren relevanteste jene vom 9. April 1997 ist, die den Titel trägt: „Maria - einzigartige Mitarbeiterin an der Erlösung“. Zu Kalvaria / Passion / Kreuzesopfer heißt es hier:

${ }^{23}$ So der status quaestionis von Andrea Villafiorita in der Zusammenfassung seiner Dissertation: Die Mitwirkung Mariens an der Erlösung. Status quaestionis der neueren Diskussion, in: FKTh 1 (2013) 2236.

${ }^{24}$ Der spezielle heilsgeschichtliche Ansatz der Mariologie von H. Köster wird behandelt in der Doktorarbeit von Stefan Hartmann. Vgl. ders., Die Magd des Herrn. Zur heilsgeschichtlichen Mariologie Heinrich Kösters (Eichstätter Studien, NF, Bd. 61), Regensburg 2009 (510 S.). Eine Besprechung der Dissertation aus der Feder von Johannes Stöhr findet sich im Mariologischen Jahrbuch 13 (2009) 122-130. Außerdem ist zu verweisen auf einen von S. Hartmann im Mariologischen Jahrbuch veröffentlichten Artikel, auf den M. Hauke kritisch repliziert hat. Vgl. S. Hartmann, Maria als „Corredemptrix / Miterlöserin“, in: Mariologisches Jahrbuch 14 (2010) 47-61; M. Hauke, Die Lehre von der Kirche über die Mitwirkung Mariens an der Erlösung - Randbemerkungen zu einer theologischen Stellungnahme, in: Mariologisches Jahrbuch 14 (2010) 63-74. 
„Die Mitwirkung Mariens [...] hat sich während des Geschehens selbst ereignet in ihrer Eigenschaft als Mutter; sie erstreckt sich folglich auf die Ganzheit des Heilswerkes Christi. Nur sie wurde auf diese Weise dem Erlösungsopfer beigesellt, welches das Heil aller Menschen verdient" ${ }^{\text {" }}{ }^{25}$ Durch ihre enge Verbindung mit Christus hat Maria auch teil an den drei Ämtern Christi, der für uns Menschen Prophet ist und Priester und König oder Hirte.

Unter den Maria zugeschriebenen Titeln ist jener der „neuen Eva" besonders geeignet, die umfassende Mittlerschaft Mariens zum Ausdruck zu bringen, weist er doch hin auf die Frau, die als mütterliche Gefährtin dem neuen Adam beim Erlösungswerk zur Seite gestellt wurde. ${ }^{26}$

Aus ihrem Handeln als Mutter und Gefährtin des Erlösers ergibt sich die geistliche Mutterschaft Mariens, in der ihre physische Mutterschaft im Hinblick auf den Sohn Gottes ihre organische Fortsetzung findet. Johannes Paul II. verschränkt die mittlerische Tätigkeit Mariens mit ihrer Mutterschaft, die das Wirken Mariens für die geistliche Geburt der Kinder Gottes hervorhebt, und spricht von der „mütterlichen Mittlerschaft Mariens in Christus".

\section{Neue Gnosis in der Mariologie: Die Ur-Maria als kosmische Matrix (Gisbert Greshake)}

In seinem umfangreichen Spätwerk zur Mariologie ${ }^{27}$ nimmt G. Greshake, was die Gnadentheologie betrifft, zunächst keinen wesentlichen Unterschied zwischen ihr und der Begnadung

25 Johannes Paul II., Marianische Katechese 48, 29.4.1997.

26 Vgl. M. Hauke, Maria als mütterliche Mittlerin, 25.

27 Vgl. G. Greshake, Maria - Ecclesia. Perspektiven einer marianisch grundierten Theologie und Kirchenpraxis, Regensburg 2014. Vgl. dazu die Besprechungen von K.-H. Menke, in: ThPh 89 (2014) 419-428; T. Marschler, in: Theologische Revue 110 (2014) 509-512; Ch. Binninger, in: AMBO 1 (2016) 394-396. 
aller Menschen an. Und im Hinblick auf die Eschatologie ist seine Deutung der Assumptio Mariens von seiner These einer Auferstehung im Tod bestimmt, deren Kerngedanken er hier wiederholt: Die Vollendung Mariens ist nur exemplarische Manifestation dessen, was allen Menschen im Tod geschieht. Aber nicht die Befassung mit den einzelnen Mariendogmen ist das Grundanliegen seines Werkes, sondern die Einführung einer neuen Vision, die Maria als urbildlich und real präexistent mit der Kirche identisch nimmt.

\subsection{Die Prädestination Mariens}

Greshake verknüpft die ewige Erwählung Mariens mit einer Präexistenzthese. Er meint die Identifikation von Maria/Ecclesia und „erstgeschöpflicher" Weisheit sei hinreichender Grund für diese These. Tatsächlich wurde die Weisheit, die in den späten Schriften des alttestamentlichen Kanons begegnet, von christlichen Theologen als Hinweis auf den präexistenten Logos verstanden. Greshake trägt die Belege für seine abweichende Deutung aus dem Buch von Th. Schipflinger ${ }^{28}$ zusammen. Es handelt sich dabei um eine wissenschaftlich nicht seriöse New-AgeProgrammschrift. Sie vermögen allesamt nicht zu überzeugen. Die allegorische Schrift-Auslegung hat zwar dazu geführt, dass die Liturgie einige Weisheitstexte an Marienfesten verwendet, doch bedeutet dies keine uneingeschränkte Identifikation. Eine Quelle für eine solche wäre indes in der russischen Sophiologie des 19./20. Jahrhunderts zu finden, auf die Greshake allerdings wenig eingeht.

Im ersten Kapitel des Hauptteils bedient sich Greshake einer glaubensphänomenologischen Herangehensweise an die Gestalt Maries. Dabei will er eine reiche Palette an Konsequenzen für die

${ }^{28}$ Vgl. T. Schipflinger, Sofia-Maria. Eine ganzheitliche Vision der Schöpfung, Oberpframmern, 2003; vgl. dazu T. Marschler, 510f. 
Neuformulierung der Struktur und des Lebens der Kirche aus seinen „allgemeinen marianischen Prinzipien“ ableiten, wobei er den Boden sicherer argumentativer Theologie verlässt. Es verwundert immer wieder, dass manche Autoren die Kirche als eine amorphe Masse ohne im Willen Christi verfügte Formbestimmtheit und Struktur begreifen, als ein menschliches Artefakte, das je neue nach der Vorgabe des Mainstreams von außen mit wechselnden Bestimmungen zu versehen ist. Auch Greshake geht es in der Zielrichtung um kirchenpolitische Konsequenzen, die auf eine Ummodelierung der Kirche in ihrer sakramentalen Struktur hinauslaufen - eine Umformung, die sich daraus ergibt, das Theologen wie er dieser bloßen Masse an kirchlichem „Urstoff“ ihre Daumen aufdrücken, um sie nach eigenem „Bild und Gleichnis" zu gestalten. ${ }^{29}$

Kapitel vier entfaltet dann die Kernthese von der primordialen vorzeitlichen Ur-Maria (Geschaffen als Anfang seiner Wege ${ }^{\text {" }}{ }^{30}$ Hier finden sich die am meisten gnostisierenden Thesen des Buches: Vf. kommt zum Postulat eines ersten Geschöpfes, das nicht nur vorzeitlich, ersterschaffen, sondern schon seit allem Anfang - vor Beginn der zeitlichen Welt - mit dem Logos vermählt ist. Im Verbund mit ihm ist ein Wechselspiel von Wort und Antwort, Gabe und Rückgabe (Gegen-Gabe) grundgelegt. Dieses prähistorische Urgeschöpf, personales oder ideelles Prinzip, ist einerseits die sapientia creata, dann wieder die ecclesia

${ }^{29}$ Die Kirche des neuen Zeitalters, die den marianischen Primat des Empfangens und der Glaubenspraxis ernst nimmt, wird, wie Greshake meint, die vorangetriebene Objektivierung und Differenzierung der Glaubensinhalte aufheben; konkret denkt er dabei an Aussagen über die Wirkung von Gnade und Sakramenten, kirchliche Amtsstrukturen, die Anzahl der Sakramente etc. Und er prophezeit für die Kirche der $\mathrm{Zu}-$ kunft „einen Konsens (consensus fidei) über die Nicht-Definierbarkeit vieler tradierter ,verbindlicher' Glaubensinhalte“. Vgl. G. Greshake, Maria-Ecclesia. Perspektiven einer marianisch grundierten Theologie und Kirchenpraxis, 407.

30 Ebd., 490-569. 
primogenita, die mit der Maria-ecclesia synonym ist. In ihr sind schon antizipativ alle Völker, Religionen, Stämme und Nationen - vorausgehend zu einer geschichtlichen Selbstmitteilung Gottes - einbehalten. Gegenüber der geschichtlich ergehenden Offenbarung Gottes und seinem Gnadenangebot ist die Urvermählung Gottes mit der Ur-Maria die alles entscheidende Wirklichkeit. Ihr kommt der Primat vor allen kontingenten Heilsereignissen zu. Greshake erblickt in seinem Theologoumenon den archimedischen Punkt einer Gnadenlehre: Innerhalb seines Denkrahmens stellt sich das alte Problem von heilsgeschichtlicher Partikularität und Universalität nicht mehr, wie nämlich ein einzelner Punkt in der Geschichte der Menschheit Ursache für die Durchsetzung des alle Menschen umfassenden Heilswillens sein soll. Man hatte ja immer gefragt: Wie kann die Inkarnation Gottes in einem einzigen Menschen - Jesus von Nazareth - an einem Punkt der Geschichte die Ursache für das universale Heil aller sein.

Greshakes Entwurf stellt, mit den Worten Th. Marschlers, den Versuch einer transzendentalen Letztbegründung für das dar, was Rahner das „übernatürliche Existential“ genannt hat. ${ }^{31}$ Es gibt eine apriorisch-gnadenhafte Erwählung aller Menschen, die in einer universalen überzeitlichen Bundestheologie fundiert wird.

Th. Marschler hebt in seiner Rezension ${ }^{32}$ des Werkes drei wesentliche Kritikpunkte hervor, die von mir in jeder Hinsicht geteilt werden: Die in diesem Werk von Greshake lancierten Hauptthesen entbehren des Rückhalts in Schrift und Tradition. Die Argumentation leidet an der Unschärfe zentraler Begriffe, aufgrund der es zu äquivoken Formulierungen und falschen Schlüssen kommt. Würde die historische Gestalt Mariens als Person ernstgenommen, so wäre ihr Gott entgegengebrachtes „Ja“ die freiwillentlich vollzogene, kontingente Antwort eines

31 Vgl. ebd., 529, vgl. T. Marschler, 511.

32 Vgl. T. Marschler, 511. 
Geschöpfes. Durch ein solches personlaes Ja-Wort könnte aber nicht die alle späteren Stellungnahmen von freien menschlichen Subjekten relativierende Unterfassung aller Freiheitsantworten konstituiert werden. Nimmt man aber umgekehrt eine prädeterminierende Gnade an, die den menschlichen Entscheidungen zuvorkommt, so wäre das „Urereignis" einer stellvertretenden Antwort des primordialen Urgeschöpfes überflüssig, denn alle späteren Freiheitsentscheidungen wären schon unter diese Prädestination gestellt. Daher trägt die These zur Lösung des Gnade-Freiheit-Problems nichts bei. Nach Greshake besitzt die von ihm angenommene „Urvermählung“ mit dem Erstgeschöpf - als primordiales Geschehen - gegenüber der Inkarnation des Gottessohnes den sachlichen Primat. D.h. die Inkarnation ist demgegenüber sekundär. Nach Greshake ist die vorgängig zu deren Glaubensantwort einsetzende „Annahme aller Menschen durch Gott" nicht bloß prä-inkarnatorisch gewollt, sondern real vollzogen. Die Geschichte mit der Kontingenz ihres Ausgangs wird entwertet, sofern sie nur noch als der „Prozess“ gilt, „in welchem das marianische Ur-Ja zur Vermählung weitergesprochen wird“. ${ }^{33}$ Nach dem Urteil Marschlers liegt in der Inanspruchnahme einer vorzeitlichen Geschichte mit einem determinierenden Urereignis ein nicht zu übersehender "Gnosis- und Mythologieverdacht“, der von Vf. nicht wirksam entkräftet werden kann.

\section{Verschiedene Autoren und ihre Forschungen}

\section{Ein exegetisches Sammelwerk}

Hans-Ulrich Weidemann (Hrsg.), "Der Name der Jungfrau war Maria” (Lk 1,27). Neue exegetische Perspektiven auf die Mutter Jesu (Stuttgarter Bibelstudien 238), Stuttgart 2018. Das Werk

${ }^{33}$ Vgl. G. Greshake, Maria - Ecclesia. Perspektiven einer marianisch grundierten Theologie und Kirchenpraxis, 527. 
entstammt einem Oberseminar mit dem inzwischen emeritierten Tübinger Exegeten Michael Theobald, der den ersten und umfangreichsten Beitrag geliefert hat. Theobald ist extrem liberal und meint, Lukas und Matthäus hätten die Jungfrauengeburt mit frei erfundenen Geschichten aus Jes 7,14 LXX extrapoliert. Das ist typisch für den Duktus des gesamten Werkes. Theobald stützt sich hermeneutisch auf D.F. Strauss, der nach wie vor lesenswert sei... ${ }^{34}$

\section{Maria als Konkretion und Verkörperung der Tochter Zion (Karl-Heinz Menke)}

K.-H. Menke deutet die Gestalt Mariens, indem er stark Bezug nimmt auf deren alttestamentliche Typologie: In Maria vergegenwärtigen und personalisieren sich die Züge der Frau Zion, die zugleich Jungfrau und Braut Jahwes genannt wird und die dem sich offenbarenden Gott - stellvertretend für ganz Israel - die Antwort des Glaubens gibt.

Im Durchgang der einschlägigen Perikopen bei Mt und Lk, aber auch bei Joh, zeigt Menke, wie sehr die Evangelisten bei dem Bild, das sie von Maria zeichnen, große Teile des AT vor Augen haben, die ihnen als Vorlage dient: Man vergleiche z. B. nur die Schilderung des Aufenthaltes der Bundeslade im Haus des Obed-Edom (2 Sam 6,8) mit dem lukanischen Bericht über den Besuch Marias bei Elisabeth. ${ }^{35}$ Menke betrachtet auch die Doppelbödigkeit vieler Aussagen des Johannesevangeliums, der eine theologische Intention zugrunde liegt. Dabei zieht er die profunden Untersuchungen des flämischen Jesuiten Ignace de la Potterie, den zu rezipieren sich die deutsche Theologie bislang

${ }^{34}$ Vgl. H.-U. Weidemann (Hrsg.), "Der Name der Jungfrau war Maria" (Lk 1,27). Neue exegetische Perspektiven auf die Mutter Jesu, Stuttgart 2018, 21.

${ }^{35}$ Vgl. K.-H. Menke, Fleisch geworden aus Maria, $35 \mathrm{f}$. 
verweigert hat, zur Vergewisserung seiner Position heran. Vf. ist sich bewusst, dass er damit neue Wege beschreitet:

„Eine Exegese, die das NT vom AT her versteht und zumindest im Johannesevangelium hinter dem Literalsinn jeder Einzelaussage eine eigentliche (theologische) Bedeutung vermutet, nimmt sich im Kontext deutscher und angelsächsischer Autoren wie ein Fremdkörper aus". ${ }^{36}$

Als Beispiel sei nur auf die Exegese von Joh 2,1-12 verwiesen, in der es augenscheinlich um eine gewöhnliche jüdische Hochzeit geht, auf einer zweiten Ebene jedoch die messianische Hochzeit Jahwes mit seiner Braut Israel zum Thema wird: Die Antwort Jesu an seine Mutter: „Was willst du von mir, Frau? Ist denn meine Stunde nicht schon gekommen" (Tatian, Ephraim d. Syrer, Gregor v. Nyssa, Theodor von Mopsuestia, Vanhoye, de la Potterie) muss mit ähnlichen Antworten beim 4. Evangelisten, welche die vordergründige Ebene einer rein äußeren Feststellung durchbrechen, verglichen werden. Dann ergibt sich - in dieser Übersetzung - für Vers Joh 2,4 eine streng messianische Konnotation, denn er enthält einen Hinweis auf die Stunde der Vollendung, das Offenbarwerden des Messias (vgl. Joh 7,39; 12,28; 17,1), die Passion des Kreuzes, an dem der Sohn in der Erneuerung des Bundes die Hochzeit mit dem Neuen Israel, der Kirche, schließt.

Der Messias wird in dieser ganzen Szene Joh 2,1-12 als der wahre Bräutigam der Braut Israel offenbar, was genau der Wein als Zeichen der endzeitlichen messianischen Freude deutlich macht - Zeichen der messianischen Güter und auch des Erscheinens des Messias. ${ }^{37}$ Schon mit der Anrede „Frau“ „transzendiert Johannes die vordergründige Ebene der Hochzeit zu Kana hin auf die messianische Ebene. Durch die Anrede ,Frau' wird Maria in Beziehung gesetzt zu der ,Frau Zion', zum Volk Israel“" ${ }^{38}$ Und

${ }^{36}$ K.H. Menke, Fleisch geworden aus Maria, 57.

37 Vgl. ebd., Anm. 69.

38 Ebd., 55. 
in der Kreuzesszene verkörpert sie das neue Sion, das neue Volk Gottes: „Die Mutter des Herrn ist das Bild der Kirche. In ihrer mütterlichen Funktion stellt sie die Kirche dar“". ${ }^{39}$

\section{Achim Dittrich: Mater Ecclesiae ${ }^{40}$}

In seiner Studie geht Achim Dittrich auf die Bedeutung des Mater-Ecclesiae-Titels auf dem II. Vatikanischen Konzil und auf dessen nachkonziliare Rezeption ein: die beiden deutschen Jesuiten Otto Semmelroth und Karl Rahner tun sich darin hervor, Maria als „Urbild der Kirche“ (Semmelroth) und „Repräsentation der Kirche" (Rahner) herauszustellen. Der Titel spielt in der postkonziliaren Liturgie eine Rolle und findet eine Wiederbelebung im Pontifikat Johannes Pauls II. In den Jahren 1979-92 findet der Mater-Ecclesiae-Titel eine durchgängige Anerkennung. Josef Ratzinger und Hans Urs von Balthasar sehen in Maria die Kirche im Ursprung: Ratzinger erblickt in ihr, der Tochter Zion, die Verkörperung der Kirche, während Balthasar die marianische Dimension der Kirche unterstreicht. Auch Benedikt XVI. greift den Titel auf.

Für das 20. Jhd. steht die Mariologie im Spannungsfeld von Ekklesiologie und Ökumene. Untersucht werden die MaterEcclesiae-Titel bei J. B. Ternien, Kardinal Mercier (Mutter der Kirche und universale Mittlerin). Dann werden verschiedene andere Zeugen gesichtet (J. Anger, Odo Casel, Feckes, Teresa Benedicta) und die Pius-Päpste, schließlich noch der Theologe Hugo Rahner. „Maria et Ecclesia“ war dann das Thema der 50er Jahre des vorigen Jahrhunderts.

Es brachte exegetische Forschungen über „die Mutter der Kirche" im johanneischen Schrifttum hervor; diesbezügliche Referate auf dem mariologischen Kongress 1950 in Rom; S. Tromp

39 Ebd., 57.

40 A. Dittrich, Mater Ecclesiae. Geschichte und Bedeutung eines umstrittenen Marientitels, (Bonner Dogmatische Studien 44), Würzburg 2009. 
war der Redakteur von Mystici Corporis. Die geistliche Mutterschaft Mariens wird damit verbunden, dass sie als „Cor Mystici Corpori“s gesehen wird. Ferner werden H. Barré, A. Müller, H. De Lubac, Th. Koehler, C. Straeter, M. Schmaus, der mariologische Kongress in Lourdes (1958) erforscht, auf dem die christotypische und ekklesiotypische Perspektive durchdiskutiert wurden. Insbesondere wird im Rückblick auf das marianische Zeitalter und im Hinblick auf das II. Vatikanum der Konnex Maria-Kirche abgefragt. Unter den Protagonisten der Mariologie auf dem II. Vatikanum werden Johannes XXIII., Kardinal Suenens, Kardinal Volk, Kardinal Bea, Gérard Philipps, C. Balic, R. Laurentin behandelt. Laurentin war immer ein entschiedener Gegner des Mater-Ecclesiae-Titels (und auch der maternitas Mariae). Interessant ist dabei eine gewisse Nähe von J. Ratzinger, von dem Dittrich feststellt, dass er „erst als Glaubenspräfekt begonnen habe, den Mater-Ecclesiae-Titel zu verwenden". ${ }^{41}$

Dittrich nennt zwei Gründe, warum der Titel auf dem Konzil nicht akzeptiert wurde: Man sah ihn einmal als Ausdruck einer alten Schule an, die es zu überwinden galt. Die moderne Theologie, die von bibeltheologischem, patristischem und ökumenischem Interesse bestimmt war, wollte nicht lehramtspositivistisch vorgehen und nicht mit dieser Marienverehrung auftreten. Zudem war das Selbstbewusstsein des Bischofskollegiums erwacht, das sich gegenüber dem Petrusamt profilieren wollte. Doch dürfe die Proklamation nicht so verstanden werden, als hätte sich der Papst gegen das Konzil gestellt.

Bei vielen internationalen Theologen findet der Titel Akzeptanz. Benedikt XVI. hat sich in seinen Äußerungen als Papst klar in die Linie von Paul VI. und Johannes Paul II. gestellt. ${ }^{42}$ Fazit des geschichtlichen Überblicks: „Der Titel Mater Ecclesiae stellt nicht ein Maria isolierendes Bildwort dar, sondern gründet

\footnotetext{
41 Vgl. ebd., 572.

${ }^{42}$ Vgl. ebd., 838.
} 
zutiefst im Mysterium der Kirche in ihrer Verbundenheit mit Christus". 43

Der Titel begegnet keineswegs nur bei „wenigen und dunklen Autoren des 12. Jahrhunderts“, wie Philipus bemerkte, sondern ist älteren Datums und schon in der Liturgie um das Jahr 1000 greifbar.

Im systematischen Teil klärt der Autor die Bedeutung von „Typus ecclesiae“ - Maria als Urbild der Kirche: Vorweg wird als Prinzip für die Mariologie der Gottesmutterschaft ein von der Sache her bestimmter Vorrang zugewiesen ${ }^{44}$; im Anschluss an Scheeben ist diese als bräutliche Gottesmutterschaft zu bestimmen. Die Bedeutung des Titels wird von Theologen der Definition von Ephesus gleichgestellt. Der Titel „Mutter" enthält die Momente Ursächlichkeit und Fürsorge.

Maria steht als Person für jene Wirklichkeit, die sich nach Pfingsten in der Kirche verwirklicht. ${ }^{45}$ Man darf den Typus-Gedanken nicht dahingehend missverstehen, das Maria zur „bloßen und damit austauschbaren Exemplifikation theologischer Sachverhalte" erklärt wird. ${ }^{46}$

Dittrich trifft in diesem Zusammenhang eine wichtige Feststellung, wenn er sagt: „Der Mater-ecclesiae-Titel hilft, den Maria-Kirche-Konnex vor einer Totalidentifizierung wie vor einer uneigentlichen Vertretung zu bewahren “ ${ }^{47}$ Maria ist nicht einfach ein Vorbild, sondern bleibendes Urbild, da sie als jungfräuliche Mutter die Kirche in Person vorweggenommen hat.

Dem Titel kommt auch eine große ökumenische Bedeutung zu. Dem Osten, so laut Maximos IV., sei der Titel unbekannt. Nach der Einschätzung mancher würde er die ökumenische Ver-

43 Vgl. ebd., 863.

44 Vgl. ebd., 876.

45 Vgl. ebd., 951.

${ }^{46}$ Vgl. J. Ratzinger, Kirche im Ursprung, 20f.

47 A. Dittrich, Mater Ecclesiae. Geschichte und Bedeutung eines umstrittenen Marientitels, 954. 
ständigung erschweren, während umgekehrt andere in Maria als der mater unitatis eine Hilfe zur Verständigung erblicken. Der Autor führt einige protestantische Stimmen zugunsten des Titels (Wickert, Wilckens) an ${ }^{48}$, benennt aber auch Erwartungen der Proklamation zugunsten eines ökumenischen Fortschritts.

Neben der Klärung des Mütterlichen verlangt der Titel auch eine ekklesiologische Klärung. Als „Tochter Zion“ veranschaulicht Maria ihre Zugehörigkeit zum Volk Israel als bester Teil und heiliger Rest, der uneingeschränkt sein Ja-Wort gegeben hat. Dittrich verfällt nicht der nach dem Konzil favorisierten Volk-Gottes-Ekklesiologie, sondern bleibt bei der für den Titel grundlegenden Leib-Christi-Sicht, wobei Maria den personalen Charakter der Kirche ausdrückt. Aber diese Kirche hat eine petrinische und marianische Prägung. Dabei veranschaulicht das marianische Moment das Prinzip der Kirche als mütterlichempfangende Gebärerin Christi, die Rezeptivität und Mitursächlichkeit des erlösungsbedürftigen Menschen. ${ }^{49}$

\section{3. „Marienrede, nicht Mariologie“ - Ivan Podgorelec“ Studie über Maria bei Karl Barth}

Bis heute wurde das Fehlen einer zufriedenstellenden Darstellung von Barths Marienrede beklagt. ${ }^{50}$ Die Frage der Mariologie betrifft das Zentrum von Barths Gnaden- und Erlösungslehre: Seine Auseinandersetzung mit der Catolica kreist um das Thema der Erlösung rein aus Gnade. Barth sieht, dass die Person und die Aktivität Mariens in enger Beziehung zur Inkarnation stehen. ${ }^{51}$ Über die Stellung Barths zu den Mariendogmen lässt sich sagen: Barth bekennt sich eindeutig zum geschichtlichen Ereignis der Jungfrauengeburt Jesu. Doch kann der Person Mari-

${ }^{48}$ Vgl. ebd., $1054 f$.

${ }^{49}$ Vgl. ebd., 1039.

${ }^{50}$ Vgl. I. Podgorelec, Marienrede, nicht Mariologie, in: FKTh 4 (2017), 289.

${ }^{51}$ Ebd., 290. 
ens aufgrund der jungfräulichen Empfängnis kein selbständiges dogmatisches Interesse zukommen. Maria ist als Jungfrau die Eingangspforte der göttlichen Offenbarung in die menschliche Welt geworden.

„Die Jungfrauschaft Mariens in der Geburt des Herrn ist die Negation nicht des Menschen vor Gott, sondern seiner Eignung, seiner Fähigkeit für Gott". ${ }^{52}$ Die Jungfräulichkeit Mariens wird von Barth nicht mariologisch aufgewertet. Sie ist bei ihm nur Zeichen des über den Menschen ergehenden Gerichts und der göttlichen Gnade. Mit der Rolle, die Maria in der Kirche innehat, hadert er. Ihr kommt eine eingeschränkte und nicht dauerhafte Aufgabe in der Heilsgeschichte zu.

Mit dem Bekenntnis zur Theotokos unterstreicht Barth mit Nachdruck die Einheit Jesu Christi, des Sohnes Mariens, mit der göttlichen Person des Sohnes Gottes. Sie ist der nur ein christologischer Hilfssatz, der nicht die Würde Mariens, sondern die ihres Sohnes beleuchtet. „Es bleibt doch die Frage, wieweit diese Gottesmutterschaft bei Barth mehr ist als nur ein biologisches Ereignis, das an der Person Mariens beziehungslos vorbeigeht ". ${ }^{.53}$ Trotzdem bildet das Fiat Mariens eine Herausforderung fürs Barths Theologie.

An sich lehnt Barth die übrigen Privilegien Mariens, die sich aus dem katholischen Dogma ergeben, ab, doch bleibt bestehen, was Pannenberg, den Podgorelec zitiert, feststellt: dass Barth sich mit seiner Erklärung der Jungfrauengeburt auf dem Weg mariologischer Reflexion befindet und dass er konsequenterweise das Dogma von der Unbefleckten Empfängnis akzeptieren müsste. ${ }^{54}$

In der katholischen Marienlehre seiht Barth Maria in einem Konkurrenzverhältnis zu Christus hervortreten. ${ }^{55}$ Barth bringt

52 Ebd., 291.

53 Ebd., 294.

54 Vgl. W. Pannenberg, Grundzüge der Christologie, Gütersloh 1964, 146.

55 Vgl. I. Podgorelec, Marienrede, nicht Mariologie, 296. 
die kirchliche und biblische Gestalt Mariens in einen Gegensatz. „Deshalb soll die kirchliche Gestalt Marias, nicht die biblische, wenn nicht ganz verschwinden, so doch wie ein Schatten werden ". ${ }^{56}$ So mündet die ganze Kritik Barths in der Behauptung einer unzulässig betonten Rolle, die Maria - wenn auch nur unselbständig - in der Heilsgeschichte spielt. Nach Podgorelec hat Barth die Mariologie nicht missverstanden, was sich in den Stellungsnahmen mehrerer Autoren dazu bestätigt. ${ }^{57}$

Es muss ein wichtiges Untersuchungsergebnis festgehalten werden: Die letzten Worte Barths in seiner - zwei Tage vor seinem Tod - gehaltenen Rede über Maria und die Mariologen dürften für den Ausgang seines Ringens mit der Mariologie entscheidend sein: „Man habe wohl diese ganze Frage der Mariologie theologisch doch noch nicht aufgearbeitet und man müsse sie auch evangelischerseits noch einmal von Anfang an durchdenken". .5

Barth ist bei seiner scharfen Kritik nicht stehengeblieben. Seine Kritik, an der Mariologie wird in späten Jahren wieder gemäßigter, bis er sie schließlich mit seiner letzten Äußerung ganz zurücknimmt. Der mariologische Minimalismus auf katholischer Seite, der die Nachkonzilsära geprägt hat, dürfte wohl kaum der Grund für dies Umschwenken gewesen sein. Die entscheidende Wende scheint in der Einsicht zu liegen, dass die Mariologie doch nicht Unrecht hat. Podgorelec schreibt darüber in seinem Kommentar: „Barth hat mit diesem kleinen Satz eigentlich seine ganze Kritik und Ablehnung der Mariologie in Frage gestellt. Dieser kleine Satz ist sozusagen Barths Revision, die Relativierung seiner eigenen Mariologiekritik und, wenn

\footnotetext{
Ebd., 297.

Vgl. ebd., 299.
}

${ }^{58}$ H. Ott, Steht Maria zwischen den Konfessionen?, Zürich 1975, 562, Anm. 1. 
wir das auf den Punkt bringen wollen, die Zurücknahme seiner ganzen Mariologiekritik“. ${ }^{59}$

\section{Markus Hofmann: Maria - die neue Eva}

In den romanischen Ländern hat es das Interesse der Forscher geweckt, dass Lumen Gentium im Marienkapitel das Eva-Maria-Motiv behandelt. Für den deutschsprachigen Raum lässt sich dies allerdings nicht sagen. So geht Markus Hofmann auf ein großes Desiderat ein, indem er das Thema von seinem Ursprung in der Väterzeit an aufgreift. Sein patrologischer Durchgang setzt bei Justin und Irenäus an, untersucht die Rezeption der Typologie im dritten Jahrhundert (Klemens von Alexandrien, Tertullian, Origenes, Gregor Thaumarturgos, Cyprian). Anknüpfungspunkte im AT werden ausfindig gemacht, außerkanonische Schriften nach Spuren für eine Eva-Maria-Typologie untersucht, schließlich wird das NT auf Ansätze für die Typologie befragt. In der Phase bis 300, in der eine ekklesiologisch akzentuierte Nova-Eva-Typologie hervortritt, werden Maria und die Kirche noch nicht zusammengedacht. Bis dahin lässt sich sagen: „Die beiden Typologien laufen nebeneinander her" ${ }^{60}$ Bei Ambrosius, den Hofmann eingehend behandelt, wird Maria zum Ecclesiae typus. Der patristische Durchgang zeigt: Die Väter des Ostens wie des Westens haben die „Nova Eva“ mariologisch und ekklesiologisch thematisiert.

Die marianischen Hymnen des Stundengebets lassen das Mittelalter in seiner Bedeutung für das Eva-Maria-Motiv hervortreten. Die Dogmatisierungsbullen von 1854 und 1950 sowie Lumen Gentium können als die bedeutsamsten Momente der jüngeren Entwicklung zum Thema angesehen werden. Hofmann

59 I. Podgorelec, Marienrede, nicht Mariologie 296.

60 M. Hofmann, Maria, die neue Eva, 357. 
referiert diese Dokumente, zusammen mit den Äußerungen der Päpste und dem Weltkatechismus.

Fazit der Studie: Die Eva-Maria-Typologie „stellt einen aus biblischem Denken erwachsenen Deutungsschlüssel für die heilsgeschichtlich einzigartige Stellung der Mutter Jesu dar“ ${ }^{\prime}{ }^{61}$

\section{Rainer Hangler: Juble, Tochter Zion}

Eine Studie zur Mariologie Joseph Ratzingers, die auf einer von Roman Siebenrock in Salzburg betreuten Dissertation zum Thema in Salzburg beruht, hat Rainer Hangler vorgelegt. ${ }^{62}$ Eine Kurzfassung der wichtigsten Ergebnisse der Doktorarbeit liegt seit einem Jahr auch als Aufsatz vor.

Das Geleitwort von Kardinal Koch ${ }^{63}$ betont die Verbindung der Mariologie Ratzingers mit dem Zweiten Vatikanischen Konzil.

In seiner Einleitung ${ }^{64}$ betont Hangler zurecht: „Ratzingers Mariologie bildet kein geschlossenes Werk; die zu diesem Thema erarbeiteten Vorträge, Predigten, Betrachtungen und Artikel liegen historisch und geographisch weit verstreut" ${ }^{\text {" } 65}$

Im Vergleich zu den bereits bekannten Quellen ist neu die Auswertung der Hörermitschrift einer Vorlesung aus dem Sommersemester 1957. ${ }^{66}$ Hangler stützt sich in seiner Arbeit vor allem auf diese frühen Überlegungen Ratzingers zur Mariologie. ${ }^{67}$

\footnotetext{
Ebd., 529.

62 R. Hangler, Juble, Tochter Zion. Zur Mariologie von Joseph Ratzinger / Benedikt XVI. (Ratzinger-Studien 9), Regensburg 2016, 336 S.

${ }^{63}$ Vgl. ebd., 11-14.

64 Vgl. ebd., 17-19.

65 Vgl. ebd., 18.

66 Vgl. ebd.

67 Die maschinenschriftlich vorliegende Mitschrift der Mariologie-Vorlesung an der Theologischen Hochschule in Freising vom Sommersemester 1957 wurde erstellt durch Josef Mühlbacher und Johannes Harrer. Vgl. R. Hangler, Juble, Tochter Zion. Zur Mariologie von Joseph Ratzinger / Benedikt XVI., $61 \mathrm{f}$.
} 
Bei der „Frage nach dem mariologischen Grundprinzip“ setzt Ratzinger ein beim „Glauben Mariens", der sie zur wahren Tochter Zion mache. ${ }^{68}$

Das Manuskript spiegelt die Frühphase der Theologie Ratzingers wieder, der sich aufgrund der exegetischen Dominanz in der Fragestellung zu der Aussage bewegen ließ, dass die Jungfrauengeburt ein Zeichen der Gnade sei und „weder asketischen... noch christologischen Sinn" habe ${ }^{69}$ - eine Aussage, die er noch in „Einführung in das Christentum" wiederholt. ${ }^{70}$

Deutlich wird vor allem, dass die enge Verwobenheit zwischen Maria und der Kirche nicht erst im Kontext des Zweiten Vatikanums das Denken Ratzingers bestimmt, sondern schon vorher. ${ }^{71}$ "Es wird auch ersichtlich, dass das in späterer Folge publizierte mariologische Werk in den Themen nicht gegen jene Inhalte steht, die in den Mitschriften berichtet werden". ${ }^{72}$

Ratzinger optiert schon früh für die Einbeziehung Marias als Urbild der Kirche in das mariologische Grundprinzip.

Wie Hangler zu Recht schreibt, möchte Ratzinger im Anschluss an die Kirchenväter beim "Ja Mariens“ einsetzen. ${ }^{73}$ Die Gottesmutterschaft Mariens und der christologische Ansatz stehe hingegen „erst am Ende und nicht am Anfang der Betrachtung". ${ }^{4}$

Angesichts der Tatsache, dass Ratzingers mariologische Vorlesung von 1957 zuerst die Beziehung zu Christus behandelt und erst dann die zur Kirche - was aus systematischer Perspektive

68 Vgl. ebd., 63f.

69 Vgl. ebd., 67.

70 Eine Zurücknahme dieser Meinung erfolge - auf Veranlassung Hans Urs von Balthasars in seiner Schrift „Tocher Zion“, Einsiedeln 1977, 50, Anm. 6.

${ }^{71}$ Vgl. R. Hangler, Juble, Tochter Zion. Zur Mariologie von Joseph Ratzinger / Benedikt XVI., 69.

${ }^{72}$ Vgl. ebd., 71.

${ }^{73}$ Vgl. ebd., 73-77.

${ }^{74}$ Vgl. ebd., 82. 
vorzuziehen ist -, stellt sich angesichts der eben genannten Deutung des „Ortes“ der Mariologie die Frage nach dem systematischen Ansatz, dem "mariologischen Grundprinzip“.

„Maria ist nicht nur Urbild, sondern auch „Mutter" der Kirche in ihrem mütterlichen Einfluss. Maria nimmt dabei teil am universalen Wirken Christi sowie des dreifaltigen Gottes. Das „Ja Mariens" wäre dabei zu verbinden mit ihrer Gottesmutterschaft. Eine Veröffentlichung der Mariologie-Mitschrift von 1957, über das von Hangler Gebotene hinaus, könnte genauere Auskunft darüber geben, wie Ratzinger die Verbindung zwischen dem "fiat" Mariens und ihrer Gottesmutterschaft beschreibt". ${ }^{75}$

Als fragwürdig erscheint aus der Retrospektive die Behauptung des frühen Ratzinger in der Mitschrift, , die Definition der Aufnahme Mariens erweise sich "nicht als historische, sondern als theologische Aussage“ (1977 in „Tochter Zion“, S. 73). ${ }^{76}$ Unter dem Stichwort „Problemkreise der Mariologie“ behandelt Hangler die „Gnadenmittlerschaft" und „Miterlöserschaft" Mariens.

Der aufmerksame Leser findet in der Mitschrift die Aussage, es gäbe aber keinen „Wesensunterschied [...] zwischen der Stellung Mariens in der Gnadenvermittlungsordnung und der Stellung der übrigen Christen “. ${ }^{77}$ Der Unterschied sei nur graduell. ${ }^{78}$ Damit werde „dem Gedanken einer Miterlöserschaft Mariens [...] eindeutig eine Absage erteilt". ${ }^{79}$

„Diese Ausführungen berücksichtigen nicht die einzigartige Mitwirkung Mariens an der Erlösung: nur Maria wirkt am Heilswerk Jesu selbst mit, das mit der Inkarnation beginnt und mit dem Opfer auf Golgota seinen Höhepunkt findet (die Mitwir-

${ }^{75}$ M. Hauke, Zur Mariologie von Joseph Ratzinger, in: FKTh 1 (2919) 50-64, hier: 54.

${ }^{76}$ Vgl. Hangler, Juble, Tochter Zion. Zur Mariologie von Joseph Ratzinger / Benedikt XVI., 192.

77 Ebd., 199.

78 Ebd., 200.

79 Vgl. ebd., 296. 
kung an der ,objektiven Erlösung'); die Mitwirkung der übrigen Christen hingegen bezieht sich auf die ,subjektive Erlösung.' Bei der objektiven Erlösung ist nicht zuletzt das Verdienst Mariens zu erwähnen, die in der zuvor empfangenen Gnade Gottes würdig wurde, die Mutter Gottes (und unsere Mutter) zu werden. Johannes Paul II. bringt diese Erkenntnis in seinen Marianischen Katechesen auf den Punkt“. ${ }^{80}$

Die Entwicklung des späteren Ratzinger, als er Präfekt der Glaubenskongregation und dann Papst war, wird in der Arbeit von Hangler zu wenig herausgestellt, so dass der Fortschritt in seiner Marienlehre ein wenig ausgeblendet erscheint:

„Der ,römische' Ratzinger (und Papst Benedikt XVI.) hat also durch einen intensiveren Kontakt mit der Weltkirche Perspektiven entdecken können, die dem ,deutschen' Professor noch verschlossen blieben. Das gilt erst recht für die Mitwirkung Mariens an der Erlösung". 81

Otto Semmelroth (S. 206) hatte der Maria als „Urbild“ der Kirche betont, aber deren geistige Mutterschaft gegenüber den übrigen Gliedern der Kirche nicht hinreichend herausgearbeitet. Ähnlich wie Heinrich Köster sieht Semmelroth Marias Mitwirkung an der Erlösung bloß rezeptiv; ihm gelingt es nicht, die aktive Mitwirkung zur Geltung zu bringen.

Bei Hangler findet sich keine Sentenz zum marianischen Lehramt Johannes Pauls II., das die mütterliche Mittlerschaft in Christus betont (dem Inhalt des dritten Teils der Enzyklika "Redemptoris Mater").

Während seins Pontifikates hat Papst Benedikt XVI. grundsätzlich eingeräumt, dass er aus der Lehre seines Vorgängers neue Impulse dazugewonnen hat: Er bekennt sich zur Lehre

${ }^{80}$ M. Hauke, Zur Mariologie von Joseph Ratzinger, 57.

${ }^{81}$ Ebd., $57 \mathrm{f}$. 
von der Miterlösung in Maria, ohne freilich den Ausdruck zu verwenden. ${ }^{82}$

Der gesamte Bereich der Mittlerschaft Mariens ist nicht auf das Thema der „Miterlösung“ eingeschränkt. Bei dem Ansatz der mütterlichen Mittlerschaft oder geistlichen Mutterschaft ist freilich die Mitwirkung Mariens an der Erlösung vorausgesetzt - ein Sachverhalt, der von Ratzinger ebenso bejaht wird wie die universale Reichweite ihrer Gnadenmittlerschaft in Christus.

Die mittlerweile gedruckten Dokumentationsbände „Insegnamenti di Benedetto XVI" würden einen Einblick in das gesamte lehramtliche Wirken des Papstes ermöglichen.

Es gibt in der diachron gelesenen Lehre von Joseph Ratzinger / Papst Benedikt durchaus einen theologischen Fortschritt, der in der Doktorarbeit Hanglers zu wenig beachtet wird. ${ }^{83}$

82 Vgl. ebd., 59: „Bei seiner Predigt im „Haus Mariens“ zu Ephesus lädt er die Christen dazu ein, den „Augenblick der Erlösung“ zu betrachten, ,in dem Maria, in der Darbringung des Opfers mit dem Sohn verbunden, ihre Mutterschaft auf alle Menschen und insbesondere auf die Jünger Jesu ausgeweitet hat" (vgl. auch S. 249). Am Fest der Schmerzen Mariens nach seinem Besuch in Bayern äußert Papst Benedikt, dass Maria auf Golgota „Anteil“ hat „an der heilbringenden Kraft des Leidens, indem sie ihr ,Fiat', ihr ,Ja', mit dem des Sohnes vereint“. Bei der Menschwerdung Gottes, so betont der Heilige Vater in Altötting, fällt das Jawort Mariens mit dem Jawort des inkarnierten Gottessohnes zum Erlösungsopfer zusammen."

83 So heißt es dort etwa, Papst Benedikt XVI. bewege sich „vollständig in den Bahnen von LG VIII“ (Lumen Gentium) (S. 273). Doch es ist zu konstatieren, dass das marianische Lehramt vor und nach dem Konzil durchaus über das hinausreicht, was expressis verbis im achten Kapitel von Lumen Gentium zu finden ist. Vgl. M. Hauke, Zur Mariologie von Joseph Ratzinger, 60: „Im päpstlichen Lehramt Pius' XII., Pauls VI. und Johannes Pauls II. finden wir hingegen Aussagen, die darüber hinaus betonen, dass Maria auch ihrerseits ihren Sohn aufopferte für die Sünden der ganzen Menschheit. Es scheint, dass Benedikt XVI. sich dieser Perspektive geöffnet hat". 


\section{Wichtige Publikationen}

\section{a. Mariologische Studien und Sedes Sapientiae}

Stefano M. Manelli, Biblische Mariologie (Mariologische Studien 27), Pustet Verlag: Regensburg 2018.

Ivan Podgorelec, Die Gestalt Mariens in Barths Marienrede. Stellung, Aufgabe und Bedeutung Mariens im Heilsplan Gottes bei Karl Barth (MSt 26), Pustet Verlag: Regensburg 2017.

Manfred Hauke (Hrsg.), Die Marienerscheinungen von Fatima - 100 Jahre danach: Geschichte, Rezeption und Bedeutung (MSt 25), Pustet Verlag: Regensburg 2017.

Manfred Hauke (Hrsg.), Maria und das Alte Testament (MSt 24), Pustet-Verlag: Regensburg 2015.

Aristide Serra, Die Frau des Bundes. Präfigurationen Marias im Alten

Testament (MSt 23), Pustet-Verlag: Regensburg 2015.

Manfred Hauke (Hrsg.), Die Herz-Mariä-Verehrung. Geschichtliche

Entwicklung und theologischer Gehalt (MSt 22), Pustet-Verlag: Regensburg 2011.

Markus Hofmann, Maria, die neue Eva. Geschichtlicher Ursprung einer Typologie mit theologischem Potential (MSt 21), Pustet-Verlag: Regensburg 2011.

Manfred Hauke (Hrsg.), Maria als Patronin Europas. Geschichtliche Besinnung und Vorschläge für die Zukunft (MSt 20), Pustet Verlag: Regensburg 2009.

Anton Ziegenaus (Hrsg.), „Geboren aus der Jungfrau Maria“. Klarstellungen (MSt 19), Pustet Verlag: Regensburg 2007.

Anton Ziegenaus (Hrsg.), Totus tuus. Maria in Leben und Lehre Johannes Pauls II. (MSt 18), Pustet Verlag: Regensburg 2004.

Manfred Hauke, Maria - „Mittlerin aller Gnaden“. Die universale Gnadenmittlerschaft Mariens im theologischen und seelsorglichen Schaffen von Kardinal Mercier (1851-1926) (MSt 17), Pustet Verlag: Regensburg 2004.

Raymund Noll, Die mariologischen Grundlinien im exegetischen Werk des Cornelius a Lapide SJ (1567-1637) (MSt 16), Pustet Verlag: Regensburg 2003. 
Gerhard Ludwig Müller, Maria - Die Frau im Heilsplan Gottes (MSt 15), Pustet Verlag: Regensburg 2002.

Anton Ziegenaus (Hrsg.), Das Marianische Zeitalter. Entstehung - Gehalt - bleibende Bedeutung (MSt 14), Pustet Verlag: Regensburg 2002.

Leo Scheffczyk, Die Mariengestalt im Gefüge der Theologie. Mariologische Beiträge (MSt 13), Pustet Verlag: Regensburg 2000.

Anton Ziegenaus (Hrsg.), Volksfrömmigkeit und Theologie. Die eine Mariengestalt und die vielen Quellen (MSt 12), Pustet Verlag: Regensburg 1998.

Achim Dittrich, Protestantische Mariologie-Kritik. Historische Entwicklung bis 1997 und dogmatische Analyse (MSt 11), Pustet Verlag: Regensburg 1998.

Anton Ziegenaus (Hrsg.), Marienerscheinungen. Ihre Echtheit und Bedeutung im Leben der Kirche (MSt 10), Pustet Verlag: Regensburg 1995. (vergriffen)

Anton Ziegenaus (Hrsg.), Maria in der Evangelisierung. Beiträge zur mariologischen Prägung der Verkündigung (MSt 9), Pustet Verlag: Regensburg 1993.

Anton Ziegenaus (Hrsg.), Maria und der Heilige Geist. Beiträge zur pneumatologischen Prägung der Mariologie (MSt 8), Pustet Verlag: Regensburg 1991.

Die Auflistung der Mariologischen Studien befindet sich auf der Internetseite des DAM. Das Mariologische Jahrbuch (Sedes Sapientiae) gibt es zum großen Teil auch im Internet: www.teol.de.

\section{b. Andere Studien}

Achim Ditrich, Mater Ecclesiae. Maria, die Mutter der Kirche. Bedeutung und Geschichte eines Marientitels, Kisslegg 2017.

Peter H. Görg, „Sagt an, wer ist doch diese“. Inhalt, Rang und Entwicklung der Mariologie in dogmatischen Lehrbüchern und Publikationen deutschsprachiger Dogmatiker des 19. Und 20. Jahrhunderts, Bonn 2007. 
Florian Kolfhaus, De Maria numquan satis. Der mariologische Ansatz des hl. Maxilimian Kolbe, in: Mar. JB 17 (2013), Bd. 2, 75-102. Michael Stickelbroeck, Die Erscheinungen von Fátima bei Edouard Dhanis s. J. - Genese und Problematik einer Kritik, in: FKTh 1 (2017) 1-20.

\section{Niemieckojęzyczna mariologia od przełomu tysiąclecia - inwentaryzacja}

W swoim przedłożeniu autor wychodzi ze spekulatywnymi próbami interpretacji tekstów nowotestamentalnych odnoszących się do Maryi i Jej zbawczej roli. Opowiada się za rozumieniem historycznym i hermeneutyką kontekstualną przeciwko ujęciu spekulatywnemu i pozbawionemu kontekstu. W tym świetle pokazuje NMP jako Pośredniczkę i Jej macierzyńskie pośrednictwo w Chrystusie. Idąc za G. Greshake, odrzuca również nowe formy gnozy w mariologii, które traktują Pra-Maryję jako kosmiczną Matrix.

W drugiej części swojego artykułu M. Stickelbroek omawia publikacje $\mathrm{z}$ ostatnich lat, jakie pojawiły się $\mathrm{w}$ świecie naukowym, a wyszły spod pióra niemieckojęzycznych teologów. I tak wskazuje na H.-U. Weidemanna, M. Hofmanna, A. Dittricha, K.-H. Menke, I. Podgoreleca, R. Hanglera oraz J. Ratzingera / Benedykta XVI. 